九州大学学術情報リポジトリ

Kyushu University Institutional Repository

Enzymatic Remodeling of Fatty Acid Molecules by SCDase Demonstrated that Fucosyl GM1a Possessing a Polyunsaturated Fatty Acid Induces Apoptosis in HL60 Human Promyelocytic Leukemia Cells

$\mathrm{Xu}, \mathrm{Xu}$

College of Life Science, Shenzhen University

Goda, Hatsumi M.

College of Life Science, Shenzhen University

Inagaki, Masanori

Graduate School of Pharmaceutical Sciences, Kyushu University

Okino, Nozomu

Laboratory of Marine Resource Chemistry, Department of Bioscience and Biotechnology, Faculty of Agriculture, Kyushu University

他

https://doi.org/10.5109/16126

出版情報: 九州大学大学院農学研究院紀要. 54 (2)，pp.433-437，2009-10-29. Faculty of Agriculture, Kyushu University

バージョン：

権利関係 : 


\title{
Enzymatic Remodeling of Fatty Acid Molecules by SCDase Demonstrated that Fucosyl GM1a Possessing a Polyunsaturated Fatty Acid Induces Apoptosis in HL60 Human Promyelocytic Leukemia Cells
}

\author{
$\mathrm{Xu} \mathrm{XU}^{1}$, Hatsumi M. GODA ${ }^{1}$, Masanori INAGAKI ${ }^{2}$, Nozomu OKINO \\ and Makoto ITO $^{3 *}$ \\ Laboratory of Marine Resource Chemistry, Department of Bioscience and Biotechnology, \\ Faculty of Agriculture, Kyushu University, Hakozaki, Higashi-ku, \\ Fukuoka 812-8581, Japan \\ (Received June 29, 2009 and accepted July 13, 2009)
}

\begin{abstract}
Glycosphingolipids show heterogeneity in carbohydrate as well as lipid (ceramide) moieties. However, the biological relevance of the heterogeneity of ceramide has yet to be elucidated. To assess the significance of fatty acid molecules of glycosphingolipids, enzymatic remodeling of fatty acyl chains of glycosphingolipids was performed using sphingolipid ceramide $N$-deacylase which catalyzes a reversible reaction in which the $N$-acyl linkage of ceramide is cleaved or synthesized. Using the hydrolysis and condensation reactions of the enzyme, fucosyl GM1a having a single but different fatty acid molecule were synthesized. We found that fucosyl GM1a having polyunsaturated fatty acids (PUFA-Fuc-GM1a) inhibited the proliferation of human leukemia-derived HL60 cells, whereas parental fucosyl GM1a did not. PUFA-Fuc-GM1a, but not lyso-Fuc-GM1a which lacks fatty acid molecules, was found to induce apoptosis in HL60 cells. This study clearly shows that the fatty acid moiety of glycosphingolipids could significantly affect their pharmacological activities.
\end{abstract}

\section{INTRODUCTION}

Glycosphingolipids (GSLs) are amphipathic compounds consisting of sugar and ceramide moieties and GSLs having sialic acids are generally named gangliosides. More than 400 species of GSLs possessing different sugar structures have been reported, although only seven monosaccharides have mainly been found in vertebrate GSLs. GSLs are found in the outer leaflet of the plasma membranes of vertebrates and thought to play functional roles in various cellular events, such as cell proliferation, cellular interaction and recognition including microbial infection (Hakomori et al., 1993; Hakomori et al., 1995). GSLs show heterogeneity not only in their carbohydrate but also in their ceramide moiety which is composed of sphingoid bases and fatty acid moieties. For example, gangliosides from bovine brain contain more than 7 species of fatty acids although stearic acid (C18:0) is a major constituent (Avrova and Zabelinskii, 1971). The structure of ceramide, especially the fatty acid moiety, could influence the localization and function of GSLs on plasma membranes, possibly by direct interaction with cholesterol and phospholipids (Bittman et al., 1994; Smaby et al., 1996). However, the biological significance of the heterogeneity of ceramide is still not well understood.

Most sphingolipids (GSLs and sphingomyelin) have saturated fatty acyl chains, while glycerophospholipids are rich in kinked unsaturated fatty acyl chains. This allows sphingolipids to pack tightly with cholesterol to

\footnotetext{
College of Life Science, Shenzhen University, Nanhai Ave 3688, Shenzhen 518060, China

Graduate School of Pharmaceutical Sciences, Kyushu University, Maidashi, Higashi-ku, Fukuoka 812-8582, Japan

Bio-architecture Center, Kyushu University, Hakozaki, Higashi-ku, Fukuoka 812-8581, Japan

* Corresponding author (E-mail: makotoi@agr.kyushu-u.ac.jp)
}

form domains in the glycerophospholipid bilayer, and provides sphingolipids with their high melting temperature (Brown et al., 2000; Dietrich et al., 2001).

Sphingolipid ceramide $N$-deacylase (SCDase) is an enzyme which hydrolyzes the $N$-acyl linkage of the ceramide moiety of various GSLs and sphingomyelin (Ito et $a l ., 1995)$. This enzyme has been found in several bacteria and cloned from the genomic library of a marine bacterium, Shewanella alga G8 (Furusato et al., 2002). SCDase also efficiently catalyzes a reverse hydrolysis reaction in which fatty acids are condensed to lyso-forms of sphingolipids (Kita et al., 2001). Using this unique reaction of SCDase, the remodeling of the fatty acid moieties of GSLs can be carried out easily with high yield (Nakagawa et al., 1999; Tani et al., 1998; Mitsutake et al., 1998; Kita et al., 2001).

Lysosphingolipids, which lack fatty acyl chains of sphingolipids, are known to be accumulated in some cases of sphingolipid storage diseases (Nakagawa et al., 2005). We have reported that lysosphingolipids including lysoGM1a induced apoptosis in mouse neuroblastoma Neuro2a cells and suggested that the accumulation of lysosphingolipids, but not parental sphingolipids, triggered the apoptotic cascade in neuronal cells of patients with sphingolipidosis (Sueyoshi et al., 2001). GM1a containing $\alpha$-linolenic acid (C18:3-GM1a) also induces apoptosis in Neuro2a cells. The fact that lyso- and polyunsaturated fatty acid (PUFA)-containing gangliosides showed strong cell toxicity may partly explains why cells do not synthesize these aberrant gangliosides as a major constituent (Nakagawa et al., 2005).

We report here that fucosyl GM1a (Fuc-GM1a) was reconstructed with a single but different PUFA using SCDase. Lyso-Fuc-GM1a and PUFA-Fuc-GM1a were found to inhibit the proliferation of HL60 human promyelocytic leukemia cells. Interestingly, PUFA-Fuc-GM1a, 
but not lyso-Fuc-GM1a, were capable of inducing apoptosis in HL60 cells. This result may indicate that the fatty acid moiety of GSLs could significantly affect their pharmacological activities.

\section{MATERIALS AND METHODS}

\begin{abstract}
Materials
Fuc-GM1a was prepared from porcine brains as described ( $\mathrm{Xu}$ et al., 2007). Recombinant SCDase of Shewanella alga G8 was also prepared as described (Furusato et al., 2002). HL60 human promyelocytic leukemia cells were obtained from Dr. H. Nojiri (Faculty of Pharmacology, Teikyo University, Japan). Stearic acid (C18:0), $\alpha$-linolenic acid (C18:3), and docosahexaenoic acid (DHA, C22:6 n-3) were obtained from Sigma (USA). Pre-coated Silica Gel 60 TLC plates were purchased from Merck (Germany). Sep-Pak Plus Silica was purchased from Waters (USA). Sphingosylphosphorylcholine (SPC, a mixture of D-erythro- and L-threo-isomers) was obtained from Sigma (USA) and Matreya (USA). RPMI 1640 medium and phosphate buffered saline (PBS) were obtained from Nissui Seiyaku Co., Ltd. The cell counting kit was from Dojindo (Japan). Trypan blue stain was from Invitrogen (USA). All other reagents were of the highest quality available.
\end{abstract}

\section{Preparation of lyso-Fuc-GM1a}

Two milligrams of Fuc-GM1a was treated with rG8 SCDase in $2.4 \mathrm{ml}$ of $25 \mathrm{mM}$ sodium acetate buffer, $\mathrm{pH} 5.5$ containing $0.5 \%$ Triton X-100, $2.5 \mathrm{mM} \mathrm{MgCl}_{2}, 2.5 \mathrm{mM}$ $\mathrm{MnCl}_{2}$, and $2.5 \mathrm{mM} \mathrm{CaCl}_{2}$. Following incubation at $37^{\circ} \mathrm{C}$ overnight, the reaction mixture was applied to the Oasis MCX cartridge. After a wash with $10 \mathrm{ml}$ of $\mathrm{MeOH}$, lysoFuc-GM1a was eluted with $6 \mathrm{ml}$ of $5 \%$ ammonia-MeOH solution. The eluate was evaporated with a Speed Vac concentrator, dissolved in distilled water and lyophilized.

\section{Synthesis and purification of the reconstructed Fuc-GM1a with a single fatty acid molecule}

One milligram of lyso-Fuc-GM1a and $5 \mu \mathrm{mol}$ of fatty acid (C18:0, C18:3 or DHA) were treated with $8 \mathrm{mU}$ of rG8 SCDase in $1.6 \mathrm{ml}$ of $25 \mathrm{mM}$ Tris- $\mathrm{HCl}$, pH 7.5, containing $0.25 \%$ Triton $\mathrm{X}-100$. Following incubation at $37^{\circ} \mathrm{C}$ for $18 \mathrm{~h}$, the reaction mixture was dried with a Speed Vac concentrator, dissolved in chloroform/methanol (2/1, $\mathrm{v} / \mathrm{v}$ ), and analyzed by TLC using chloroform/methanol/ $0.02 \% \mathrm{CaCl}_{2}(5 / 4 / 1, \mathrm{v} / \mathrm{v})$ as a developing solvent. The synthesized Fuc-GM1a and remaining lyso-Fuc-GM1a were visualized with orcinol- $\mathrm{H}_{2} \mathrm{SO}_{4}$. The mixture was applied to the Oasis MCX cartridge. After a wash with $10 \mathrm{ml}$ of MeOH, lyso-Fuc-GM1a was washed out with $6 \mathrm{ml}$ of $5 \%$ ammonia-MeOH solution. The fractions containing the reconstructed Fuc-GM1a were pooled, evaporated with a Speed Vac concentrator and applied to a Sep-Pak Plus Silica cartridge. After the cartridge was washed with $60 \mathrm{ml}$ of chloroform/methanol $(3 / 1, \mathrm{v} / \mathrm{v})$, the reconstructed Fuc-GM1a was eluted with $20 \mathrm{ml}$ of chloroform/ methanol (2/1, v/v), evaporated with a Speed Vac concentrator and used as the substrate (Xu et al., 2007).
Analysis of fatty acid composition analysis and fast atom bombardment mass spectrometry (FAB-MS)

For analyzing fatty acid composition, 50 nanomoles of the reconstructed Fuc-GM1a was determined by gas chromatography (GC-14A, Shimadzu Co., Japan) as described (Xu et al., 2007). Ten micrograms of the reconstructed Fuc-GM1a was mixed with triethylene glycol and hexamethylphosphoric triamide as a matrix and then subjected to FAB-MS using a Jeol SX102A mass spectrometer used in the negative ion mode (Jeol, Japan) (Xu et al., 2007).

\section{Cell culture}

HL60 cells were cultured in RPMI 1640 medium supplemented with $60 \mathrm{mg} / \mathrm{L}$ of kanamycin and 10\% FBS at $37^{\circ} \mathrm{C}$ in an atmosphere of $95 \%$ air and $5 \% \mathrm{CO}_{2}$.

\section{Assay of cell viability by a cell counting kit}

HL60 cells $\left(5 \times 10^{5}\right)$ were treated with $40 \mu \mathrm{M}$ FucGM1a, lyso-Fuc-GM1a and PUFA-Fuc-GM1a at $37^{\circ} \mathrm{C}$ in RPMI 1640 medium supplemented with $2 \%$ FBS in the wells of 96-well plates for $24 \mathrm{~h}$, then the viability of the cells was evaluated by using a cell counting kit (Dojindo). The absorbance was measured using a microplate reader set at $490 \mathrm{~nm}$.

\section{Assay of cell lethality by trypan blue staining}

HL60 cells $\left(5 \times 10^{5}\right)$ were treated with $40 \mu \mathrm{M}$ FucGM1a, lyso-Fuc-GM1a and PUFA-Fuc-GM1a at $37^{\circ} \mathrm{C}$ in RPMI 1640 medium supplemented with 2\% FBS in the wells of 96-well plates for $24 \mathrm{~h}$. Then the cells were stained by trypan blue. The numbers of cells were counted using a hemocytometer. Cell lethality was calculated as follows: cell lethality $(\%)=$ (numbers of stained cells) $\times 100 /$ (numbers of stained cells + numbers of unstained cells).

\section{Apoptotic morphology}

HL60 cells $\left(1 \times 10^{6}\right)$ were treated with $40 \mu \mathrm{M}$ FucGM1a, lyso-Fuc-GM1a and PUFA-Fuc-GM1a at $37^{\circ} \mathrm{C}$ in RPMI 1640 medium supplemented with $2 \%$ FBS in the wells of a 6 -well plate for $24 \mathrm{~h}$. Then the cells were directly observed by microscope (TMS-F, Nikon, Japan).

\section{Flow cytometric analysis}

HL60 cells $\left(1 \times 10^{6}\right)$ were treated with $40 \mu \mathrm{M}$ FucGM1a, lyso-Fuc-GM1a and PUFA-Fuc-GM1a at $37^{\circ} \mathrm{C}$ in RPMI 1640 medium supplemented with 2\% FBS in the wells of a 6 -well plate. After incubation for $24 \mathrm{~h}$, the cells were washed, fixed with $70 \%$ ethanol, and stained with propidium iodide (PI). A flowcytometric analysis was performed with EPICS XL System II (Beckman Coulter, USA).

\section{RESULTS}

Condensation of various fatty acids to lyso-FucGM1a by SCDase and identification of the reconstructed Fuc-GM1a

Fuc-GM1a with a different fatty acid molecule was 
prepared by the condensation reaction of SCDase using lyso-Fuc-GM1a and the corresponding fatty acid. The efficiency of synthesis of Fuc-GM1a reached more than 80\% when stearic acid (C18:0), $\alpha$-linolenic acid (C18:3), and docosahexaenoic acid (DHA, C22:6 n-3) were used as a substrate (data not shown). After the purification of the reconstructed Fuc-GM1a, as described in MATERIALS AND METHODS, their structures were examined by gas chromatography and FAB-MS using the negative-ion mode. As a result, it was confirmed that each Fuc-GM1a contained the expected single fatty acid
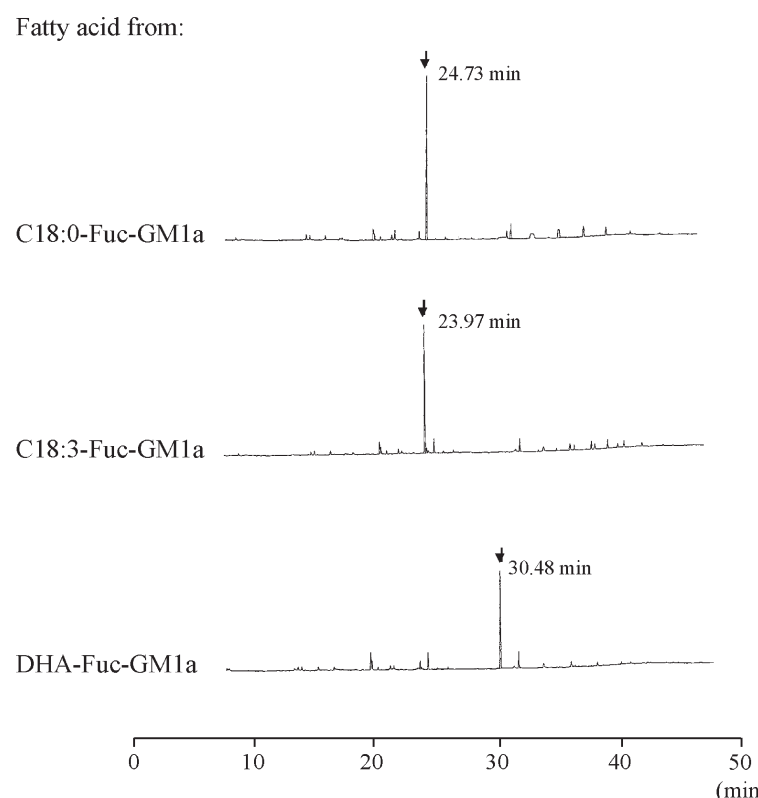

Fig. 1. Gas chromatographic analysis of fatty acid moieties of the reconstructed Fuc-GM1a.

Fatty acid methyl esters of reconstructed Fuc-GM1a were analyzed with gas chromatography, and identified using the retention time of standard fatty acids. C18:0-FucGM1a, Fuc-GM1a with stearic acid; C18:3-Fuc-GM1a, Fuc-GM1a with $\alpha$-linolenic acid; DHA-Fuc-GM1a, FucGM1a with DHA.
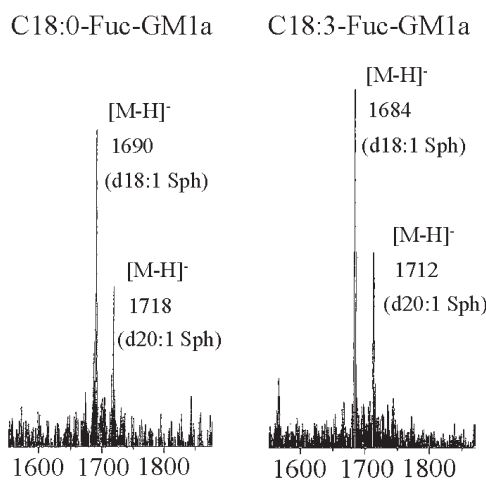

DHA-Fuc-GM1a

160017001800

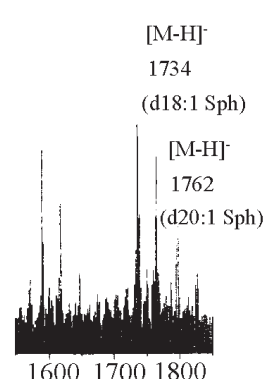

160017001800

Fig. 2. FAB-MS analysis of the reconstructed Fuc-GM1a. FAB-MS was conducted with a Jeol SX102A mass spectrometer in the negative ion mode. C18:0-Fuc-GM1a, Fuc-GM1a with stearic acid; C18:3-Fuc-GM1a, Fuc-GM1a with $\alpha$-linolenic acid; DHA-Fuc-GM1a, Fuc-GM1a with DHA.

molecule (Fig. 1). Characteristic pseudomolecular ions $[\mathrm{M}-\mathrm{H}]^{-}$were found at values of the expected molecular masses corresponding to the reconstructed Fuc-GM1a with a different fatty acid (Fig. 2). These results indicated that unsaturated fatty acids were successfully condensed by the SCDase to the lyso-Fuc-GM1a generating a Fuc-GM1a having each unsaturated fatty acid.

\section{Growth inhibition of HL60 cells by lyso-Fuc-GM1a and PUFA-Fuc-GM1a}

The addition of PUFA-Fuc-GM1a and lyso-FucGM1a to the culture of HL60 cells was found to inhibit cell proliferation. The inhibitory effects of C18:3-Fuc-GM1a and DHA-Fuc-GM1a on cell viability were stronger than those of lyso-Fuc-GM1a (Fig. 3A). On the other hand, the parental Fuc-GM1a, which contains stearic acid as the major fatty acid, did not inhibit cell proliferation, but rather stimulated cell growth compared to the control which contained no gangliosides (Fig. 3A). The numbers of dead cells were examined by trypan-blue staining. The numbers increased with the addition of lyso-FucGM1a and PUFA-Fuc-GM1a to the culture, while parental Fuc-GM1a did not affect the cell viability. It is worth noting that C18:3-Fuc-GM1a and DHA-Fuc-GM1a were much more lethal than lyso-Fuc-GM1a (Fig. 3B).

(A)

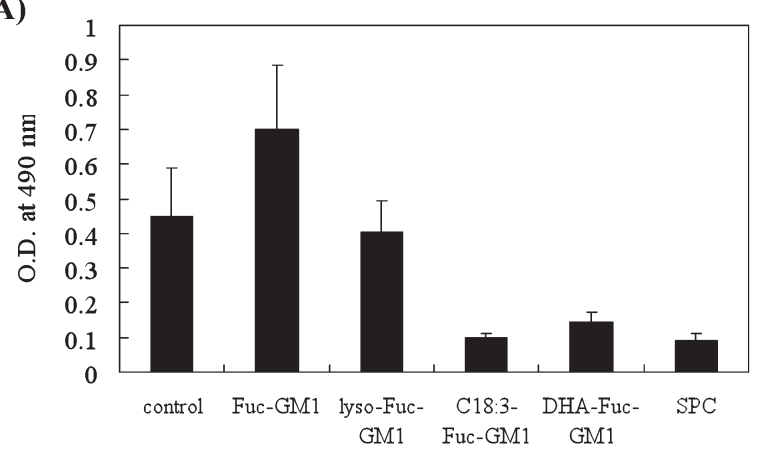

(B)

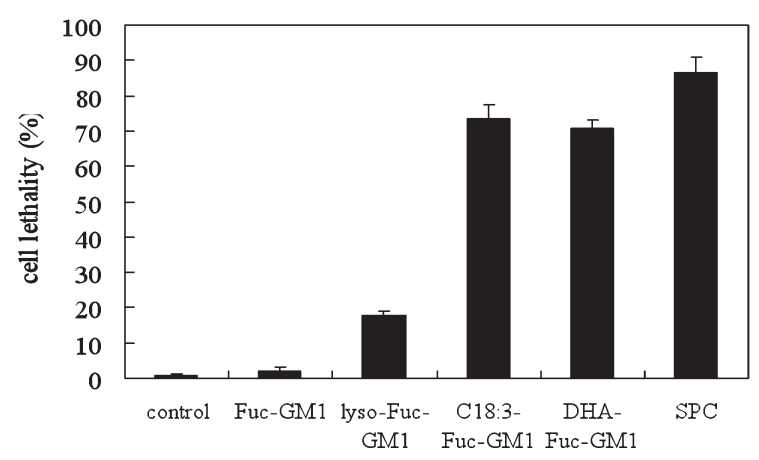

Fig. 3. Viability (A) and lethality (B) in HL60 cells treated with Fuc-GM1a, lyso-Fuc-GM1a and PUFA-Fuc-GM1a. HL60 cells $\left(5 \times 10^{5}\right)$ were treated with $40 \mu \mathrm{M}$ Fuc-GM1a, lyso-Fuc-GM1a and PUFA-Fuc-GM1a at $37^{\circ} \mathrm{C}$ for $24 \mathrm{~h}$ in RPMI 1640 medium supplemented with $2 \%$ FBS. (A), the viability of the cells was evaluated using a cell counting kit (Dojindo) as described in MATERIALS AND METHODS. O.D., optical density. (B), the cells were stained by Trypan blue. Cell lethality was calculated as described in MATERIALS AND METHODS. 


\section{Features of cell death induced by PUFA-Fuc-GM1a}

It was reported that lyso-GM1a induced apoptosis in mouse neuroblastoma Neuro2a cells (Nakagawa et al., 2005). Thus, the possibility that lyso-Fuc-GM1a and PUFA-Fuc-GM1a induces apoptosis in HL60 cells was examined. After treatment with C18:3-Fuc-GM1a and DHA-Fuc-GM1a, chromatin condensation and nuclear fragmentation, both typical features of apoptosis, were observed in HL60 cells (Fig. 4, data not shown). Interestingly, the addition of lyso-Fuc-GM1a did not result in the chromatin condensation or nuclear fragmentation in HL60 cells (Fig. 4). The apoptosis induced by PUFA-Fuc-GM1a was also quantified with a flow cytometer. A flow cytometric analysis was then performed using HL60 cells treated with gangliosides after staining with PI. The propotion of apoptotic cells reached $37 \%$ and $31 \%$ after treatment with C18:3-FucGM1a and DHA-Fuc-GM1a for $24 \mathrm{~h}$, respectively (Fig. $5 \mathrm{D}$ and $\mathrm{E}$ ), whereas it was less than $1 \%$ and $4 \%$ with Fuc-GM1a (Fig. 5B) and lyso-Fuc-GM1a (Fig. 5C), respectively. These results clearly indicated that treatment with PUFA-Fuc-GM1a (C18:3-Fuc-GM1a and DHA-Fuc-GM1a), but not parental Fuc-GM1a (C18:0Fuc-GM1a) or lyso-Fuc-GM1a, induced apoptosis in HL60 cells.

\section{DISCUSSION}

We have developed a new method of preparing
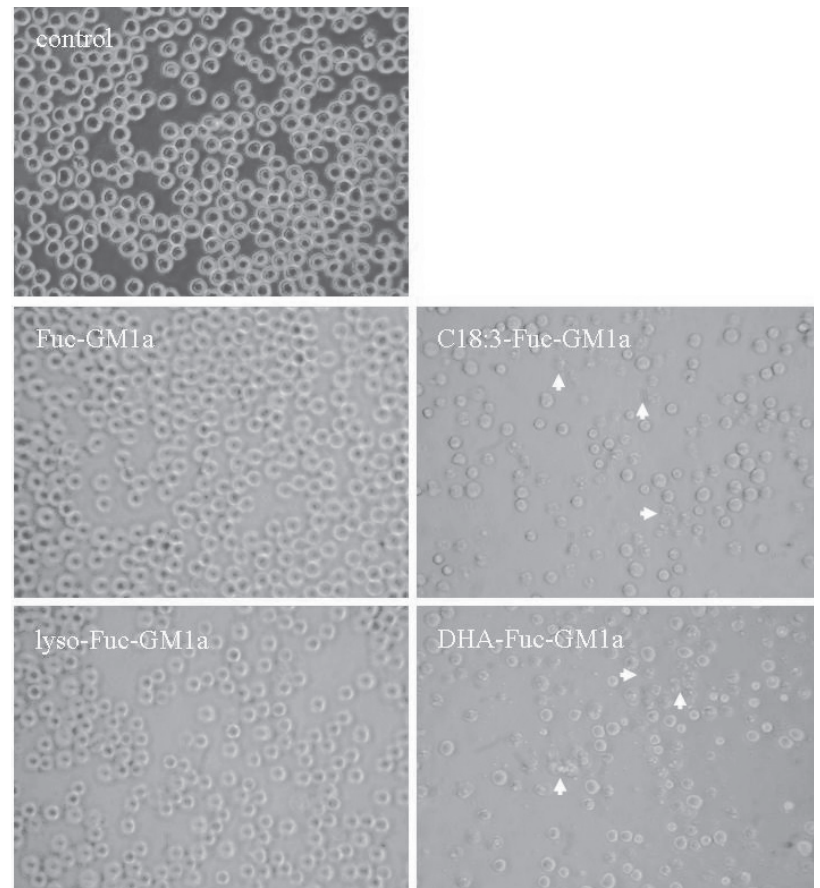

Fig. 4. Morphological change of HL60 cells induced by PUFAFuc-GM1a.

HL60 cells $\left(1 \times 10^{6}\right)$ were treated with $40 \mu \mathrm{M}$ Fuc-GM1a, lyso-Fuc-GM1a and PUFA-Fuc-GM1a at $37^{\circ} \mathrm{C}$ for $24 \mathrm{~h}$ in RPMI 1640 medium supplemented with 2\% FBS. The morphology of cells was observed under a microscope (TMS-F, Nikon, Japan).
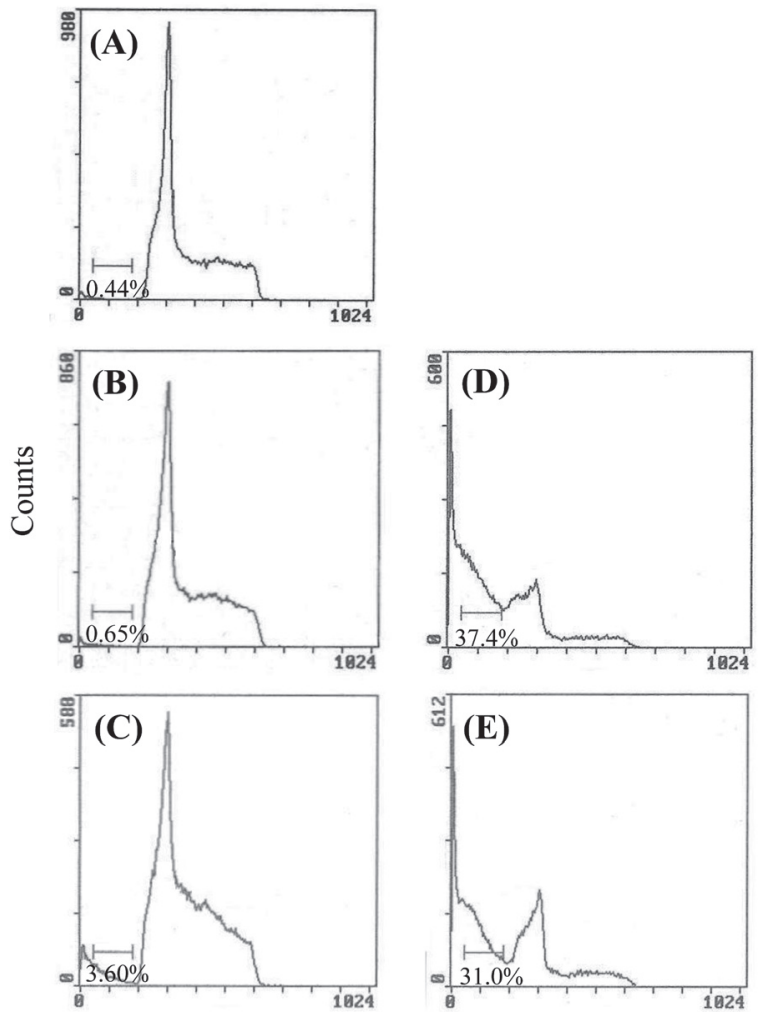

Fluorescence

Fig. 5. Quantification of apoptosis in HL60 cells with a flow cytometer

HL60 cells $\left(1 \times 10^{6}\right)$ were treated with $40 \mu \mathrm{M}$ Fuc-GM1a (B), lyso-Fuc-GM1a (C), C18:3-Fuc-GM1a (D) and DHA-Fuc-GM1a (E), respectively, at $37^{\circ} \mathrm{C}$ for $24 \mathrm{~h}$ in RPMI 1640 medium supplemented with 2\% FBS. The cells were washed, fixed with $70 \%$ ethanol, and stained by propidium iodide (PI). The DNA fragmentation was quantified using a flow cytometer. Numbers represent the percentages of apoptotic cells. Control (A) represents the percentage of apoptotic cells without treatment with gangliosides after $24 \mathrm{~h}$.

Fuc-GM1 from porcine brain using endoglycoceramidase (EGCase) (Xu et al., 2007). Fuc-GM1a, a minor component of porcine brain, is not easy to separate from the major component GM1a, by column chromatography. We found that Fuc-GM1a was resistant to hydrolysis by EGCase while other gangliosides including GM1a were easily hydrolyzed by the enzyme. Thus, Fuc-GM1a was concentrated after EGCase treatment of crude ganglioside fractions. Using the specificity of EGCase, a large amount of Fuc-GM1a can be prepared from porcine brain. Fuc-GM1a was found to be aberrantly expressed on small cell lung cancer carcinoma (SCLC) cells and thus thought to be a possible target for immunotherapy (Livingston et al., 2005). Actually, the vaccination of SCLC patients with Fuc-GM1a conjugated to keyhole limpet hemocyanin was found to induce an IgM antibody response against Fuc-GM1a and tumor cells expressing Fuc-GM1a (Krug et al., 2004). While Fuc-GM1a is similar in structure to GM1a, its physiological and pharmacological functions seem to be different.

Gangliosides usually contain saturated fatty acids not 
PUFAs in the ceramide moiety. However, some reports indicated that PUFAs are widely distributed in gangliosides as a minor constituent (Weinstein et al., 2005). Nevertheless, little attention has been paid to the physiological significance of PUFA-containing gangliosides. In this context, the physiological and pharmacological functions of PUFA-containing gangliosides should be further clarified.

To address the significance of the fatty acid moiety of GSLs, a specific and convenient method of reconstructing GSLs with unsaturated fatty acids is still required. For a long time, the only choice was a chemical procedure that could generate by-products and thus seems not to be suitable for the reconstruction of highly polarized sphingolipids, such as gangliosides (Kamio et al., 1992; Morigaki et al., 1998). SCDase, an enzyme capable of hydrolyzing the $N$-acyl linkage between fatty acids and ceramide in various GSLs and sphingomyelin (Ito et al., 1995), was found to catalyze the reverse reaction, in which a fatty acid is condensed to a lyso-GSL to yield a GSL (Kita et al., 2001). Cloning of SCDase from Shewanella alga G8 revealed that the reversible reactions were catalyzed by a single protein (Furusato et al., 2002). The present method using SCDase is much superior to the previous chemical method, because SCDase is specific to sphingolipids and capable of acting on all sphingolipids tested so far. Namely, GSLs including neutral and acid GSLs, sphingomyelin, and ceramide can be reconstructed with the desired fatty acid molecules with no by-products and at high yield. The remodeling of the fatty acid moieties of sphingolipids by SCDase will facilitate the further development of sphingolipids with new functions and GSL research.

\section{REFERENCES}

Avrova N. F. and S. A. Zabelinskii 1971 Fatty acids and long chain bases of vertebrate brain gangliosides. J. Neurochem., 18: $675-681$

Bittman R., C. R. Kasireddy, P. Mattjus and J. P. Slotte 1994 Interaction of cholesterol with sphingomyelin in monolayers and vesicles. Biochemistry, 33: 11776-11781

Brown D. A. and E. London 2000 Structure and function of sphingolipid-and cholesterol-rich membrane rafts. J. Biol. Chem., 275: 17221-17224

Dietrich C., Z. N. Volovyk, M. Levi, N. L. Thompson and K. Jacobson 2001 Partitioning of Thy-1, GM1, and cross-linked phospholipid analogs into lipid rafts reconstituted in supported model membrane monolayers. Proc. Natl. Acad. Sci. U. S. A., 98: 10642-10647

Furusato M., N. Sueyoshi, S. Mitsutake, K. Sakaguchi, K. Kita, N. Okino, S. Ichinose, A. Omori and M. Ito 2002 Molecular cloning and characterization of sphingolipid ceramide $N$-deacylase from a marine bacterium, Shewanella alga G8. J. Biol. Chem., 277: 17300-17307
Hakomori S. and Y. Igarashi 1993 Gangliosides and glycosphingolipids as modulators of cell growth, adhesion, and transmembrane signaling. Adv. Lipid Res., 25: 147-162

Hakomori S. and Y. Igarashi 1995 Functional role of glycosphingolipids in cell recognition and signaling. J. Biochem. (Tokyo), 118: 1091-1103

Ito M., T. Kurita and K. Kita 1995 A novel enzyme that cleaves the $N$-acyl linkage of ceramides in various glycosphingolipids as well as sphingomyelin to produce their lyso forms. $J$. Biol. Chem., 270: 24370-24374

Kamio K., S. Gasa and A. Makita 1992 Galactosylceramide containing omega-amino-fatty acids: preparation, characterization and sulfotransferase acceptor. J. Lipid Res., 33: $1227-1232$

Kita K., T. Kurita and M. Ito 2001 Characterization of the reversible nature of the reaction catalyzed by sphingolipid ceramide $\mathrm{N}$-deacylase. A novel form of reverse hydrolysis reaction. Eur. J. Biochem., 268: 592-602

Kurug, L. M. G. Ragupathi, and C. Hood 2004 Vaccination of patients with small-cell lung cancer with synthetic fucosyl GM1-conjugated to keyhole limpet hemocyanin. Clinic Cancer Res. 10: 6094-6100

Livingston P. O., C. Hood, and L. M. Krung 2005 Selection of GM2, fucosyl GM1, globo $\mathrm{H}$ and polysialic acid as targets on small cell lung cancers for antibody mediated immunotherapy. Cancer Immunol. Immunother., 54: 1018-1025

Mitsutake S., K. Kita, T. Nakagawa and M. Ito 1998 Enzymatic synthesis of ${ }^{14} \mathrm{C}-$ glycosphingolipids by reverse hydrolysis reaction of sphingolipid ceramide $N$-deacylase: detection of endoglycoceramidase activity in a seaflower. J. Biochem. (Tokyo), 123: 859-863

Morigaki E., M. Nagao, Y. Miura, K. Takahata, M. Tada, S. Nakajima, N. Baba and S. Shimizu 1998 Synthesis of sphingomyelins and ceramides bearing a docosahexaenoyl or arachidonoyl group. Biosci. Biotechnol. Biochem., 62: 2070-2072

Nakagawa T., A. Morotomi, M. Tani, N. Sueyoshi, H. Komori and M. Ito 2005 C18:3-GM1a induces apoptosis in Neuro2a cells: enzymatic remodeling of fatty acyl chains of glycosphingolipids. J. Lipid Res., 46: 1103-1112

Nakagawa T., M. Tani, K. Kita and M. Ito 1999 Preparation of fluorescence-labeled GM1 and sphingomyelin by the reverse hydrolysis reaction of sphingolipid ceramide $N$-deacylase as substrates for assay of sphingolipid-degrading enzymes and for detection of sphingolipid-binding proteins. J. Biochem. (Tokyo), 126: 604-611

Smaby J. M., M. Momsen, V. S. Kulkarni and R. E. Brown 1996 Cholesterol-induced interfacial area condensations of galactosylceramides and sphingomyelins with identical acyl chains. Biochemistry, 35: 5696-5704

Sueyoshi N., T. Maehara and M. Ito 2001 Apoptosis of Neuro2a cells induced by lysosphingolipids with naturally occurring stereochemical configurations. J. Lipid Res., 42: 1197-1202

Tani M., K. Kita, H. Komori, T. Nakagawa and M. Ito 1998 Enzymatic synthesis of omega-amino-ceramide: preparation of a sensitive fluorescent substrate for ceramidase. Anal. Biochem., 263: 183-188

Weinstein, D. B., J. B. Marsh, M. C. Glick, and L. Warren 1970 Membranes of animal cells. VI. The glycolipids of the L cell and its surface membrane. J. Biol. Chem. 245: 3928-3937

$\mathrm{Xu}$ X., H. Monjusho, M. Inagaki, Y. Hama, K. Yamaguchi, K. Sakaguchi, M. Iwamori, N. Okino and M. Ito 2007 Fucosyl GM1a: an endoglycoceramidase-resistant ganglioside of porcine brain. J. Biochem. (Tokyo), 141: 1-7 